\section{Psicología IBEROAMERICANA}

\section{Psicología lberoamericana}

ISSN: 1405-0943

psicología.iberoamericana@uia.mx

Universidad Iberoamericana, Ciudad de

México

México

Xelhuantzi Santillán, Rafael Izcóatl; Flores Palacios, Fátima Niño de calle: representación social del concepto en Guadalajara y Ciudad de México

Psicología Iberoamericana, vol. 22, núm. 2, julio-diciembre, 2014, pp. 54-63

Universidad Iberoamericana, Ciudad de México

Distrito Federal, México

Disponible en: http://www.redalyc.org/articulo.oa?id=133938134007

Cómo citar el artículo

Número completo

- Más información del artículo

Página de la revista en redalyc.org

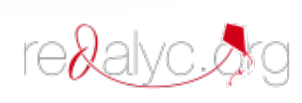

Sistema de Información Científica Red de Revistas Científicas de América Latina, el Caribe, España y Portugal Proyecto académico sin fines de lucro, desarrollado bajo la iniciativa de acceso abierto 


\title{
Niño de calle: representación social del concepto en Guadalajara y Ciudad de México
}

\author{
Street Children: Social Representation of the Concept in Guadalajara \\ and Mexico City
}

\author{
Rafael Izcóatl Xelhuantzi Santillán*1 \\ FACULTAD de Psicología \\ Universidad Nacional Autónoma de México \\ Fátima Flores Palacios** \\ Centro Peninsular en Humanidades y Ciencias Sociales
}

\section{RESUMEN}

La presente investigación exploró la "representación social" (Rs) que existe sobre el objeto social "niño de calle" en dos ciudades con contextos históricos y sociales diferentes, con el principal objetivo de distinguir las creencias, emociones y atributos que se construyen como núcleo central, y aquellos elementos de contraste, así como explorar y describir las consecuencias del concepto que circunscribe un fenómeno social mucho más complejo. Participaron un total de 110 sujetos, 56 de la Ciudad de México y 54 de Guadalajara, que fueron seleccionados por la cercanía con el fenómeno del callejerismo. Los resultados mostraron la existencia de cuatro elementos que constituyen el núcleo central de la Rs del "niño de calle": personas que viven en la calle, pobreza, tristeza y sin familia; de igual manera, hay una serie de creencias y evocaciones respecto a la figura social que son específicas de cada contexto. Estos resultados ponen en evidencia tanto la complejidad de la formación de un concepto cargado de estereotipos y prejuicios, así como la influencia e importancia que tienen los factores sociales y culturales en la representación social de un objeto.

Palabras clave: exclusión social, poblaciones callejeras, niño de calle, representación social, discriminación.

\section{ABSTRACT}

This research examines the "social representation" in relation to "street children" in two cities with different social and historic contexts, with the core aim of distinguishing between the beliefs, emotions and attributes constructed as a central nucleus, as well as contrasting elements and exploring the consequences of the concept that circumscribes a far more complex social phenomenon. The study population consisted of a total of 110 subjects -56 from Mexico City, and 54 from the city of Guadalajara- who were selected on the basis of their proximity to the phenomenon of homelessness. The results showed that four elements constitute the central nucleus of the SR of "street children": people living on the street, in poverty, in wretched conditions and without family; there is also a series of beliefs and suggestions about the social aspect that are specific to each context. These results reveal both the complexity of the formation of a concept that is highly charged with stereotypes and prejudices, as well as the influence and importance of social and cultural factors on the social representation of an object.

Keywords: Social Exclusion, Homeless Populations, Street Children, Social Representation, Discrimination.

Recibido: 20 de febrero de 2014 - Aceptado: 30 de agosto de 2014.

${ }^{1}$ Para correspondencia electrónica: izcoatl.xelhuantzi@gmail.com; fatflor@servidor.unam.mx

*Licenciado en Psicología por la UNAM, actualmente alumno del programa de Posgrado de Doctorado en Psicología en la UNAM.

** Licenciada en Psicología por la UnAM, doctora en Psicología Social por la Universidad Autónoma de Madrid, miembro del Sistema Nacional de Investigadores, profesora titular C del Centro Peninsular en Humanidades y Ciencias Sociales, sede foránea de la UNAM. 


\section{INTRODUCCIÓN}

Se puede decir con ciertas reservas, que el mundo nunca supo tanto de sí mismo y su naturaleza como hoy en día. Aun así, la lógica multicultural del capitalismo global actual, las políticas neoliberales, la acumulación de poder en pequeños sectores y, como eje fundamental, la organización de la vida social respecto al valor que se produce han acelerado el crecimiento desmedido del nivel de desigualdad social (Galende, 2011; Osorio \& Victoriano, 2011). En la actualidad, existen aproximadamente mil millones de personas en todo el mundo viviendo en pobreza extrema, según datos del World-Bank (2014), cerca de una séptima parte de la población total del planeta está inmersa en una acumulación de procesos confluyentes con rupturas sucesivas que los van arrancando del corazón de la economía, la política y la sociedad, los alejan e "inferiorizan" respecto a los centros de poder, recursos y valores dominantes, es decir, viven un proceso de "exclusión social" (Rizo, 2006).

Actualmente, la expulsión de niños, jóvenes y adultos por parte de las instituciones familiares, sociales y gubernamentales hacia las calles de las ciudades del mundo es una de las más claras y radicales muestras de exclusión social moderna, debido a que esos sujetos se encuentran por debajo del mínimo posible para acceder a los servicios, opciones o recursos (Ossa \& Lowick-Russell, 2009; Shaw, 2002; Taracena, 2010), que, según Pérez (2003), se traducen en discriminación por condición social, apariencia y por grupos de pertenencia, la insuficiencia de recursos propios y la carencia de capacidades adecuadas son los factores para la reproducción y el agravamiento persistentes de la situación de pobreza.

Se debe aclarar que, si bien las "poblaciones callejeras" ${ }^{2}$ no son un fenómeno específico de la sociedad y del sistema económico actual, sí lo es la exposición y prácticas gubernamentales e institucionales que

\footnotetext{
${ }^{2}$ La población callejera es un grupo social diverso conformado por niñas, niños, personas jóvenes, mujeres, familias, personas adultas mayores, personas con discapacidad o con diversos problemas de salud, y adicciones. En general, el término se refiere a toda persona o grupo de personas -con o sin relación entre sí-, que subsisten en la calle o en el espacio público utilizando recursos propios y precarios para satisfacer sus necesidades elementales. El término "poblaciones callejeras" se usa para nombrar "a quienes comparten la misma red social de sobrevivencia y en conjunto, han gestado una cultura callejera, es decir, de 'callejerismo"' (CDHDF, 2010).
}

las rodean. Es así que desde la década de los setenta surge un gran interés y preocupación por una de las caras de mayor exposición "mediática" del fenómeno: miles de instituciones, cientos de investigaciones científicas y decenas de organizaciones se generan respecto y alrededor de tanto niño y joven que se encuentra sobreviviendo o subsistiendo en las calles de las grandes ciudades del mundo; los esfuerzos se centran en el objetivo de su reintegración, rehabilitación y asistencia (Pérez, 2003; Makowski, 2011; Pérez \& Arteaga, 2009; Strickland, 2012).

Contrario a los objetivos principales -consensuados e idealizados por parte de la sociedad en contra de la crudeza que representa la radical exclusión social que significa subsistir en las calles-, las múltiples intervenciones acarrearon de manera involuntaria serias repercusiones al fenómeno; quizá la que más daño causó al abordaje fue la normativización, generalización y difusión del concepto "niños de calle", pues más que convertirse en una variable categórica, sirvió para estigmatizar y homogeneizar esta realidad compleja $y$, con ello, justificar múltiples prejuicios que constituyen hoy en día uno de los mayores obstáculos en el ámbito social (CDHDF, 2010; Maia \& Alves, 2004; Pérez, 2003; Urcola, 2011). En coincidencia con el señalamiento que establecen Osorio \& Victoriano (2011), no se debe exagerar si se dice que la vida útil de los conceptos depende más del efecto de resonancia que adquieren en la jerga burocrática o en los comités de las organizaciones internacionales, que en la capacidad explicativa que puedan alcanzar en los saberes académicos y en las aulas universitarias. Estos conceptos, la mayoría de las veces, están dotados de una sacralidad incuestionable, la cual trasciende las investigaciones y se instaura en la cotidianidad para perpetuar estereotipos y justificar prejuicios.

En el año de 1992, la UNICEF establece el concepto "niño de la calle", el cual definió, delimitó y homogeneizó a todo un complejo fenómeno callejero, como:

Persona menor de 18 años, desde recién nacida hasta los 17 años de edad, que sobrevive de su trabajo en la calle, que ha abandonado a su familia para evitar maltrato e incomprensión, es miembro de una red callejera de la cual padece y aprende el uso de la violencia; así como también goza de la máxima libertad 
que la misma ciudad le proporciona (Garza, 2009; Llorens et al., 2005; Lucchini, 1996).

Al ser adoptada a escala mundial, esta definición comenzó a borrar las delicadas líneas del enorme mosaico que diferenciaba la compleja heterogeneidad del fenómeno del callejerismo, así como sus características estructurales, causales, étnicas, fisiológicas, históricas y contextuales, sólo por mencionar algunas. Como ejemplo, niño(a)s, jóvenes que trabajan, viven o pasan la mayor parte del tiempo en la calle; niño(a)s, jóvenes migrantes, desplazados de guerra, entre otros tantos grupos fueron circunscritos en su mayoría en el oxímoron "niño de calle", término que simbólica, emocional y socialmente tiene una serie de repercusiones en la identidad individual y social (Llorens et al., 2005; CDHDF, 2010; Urcola, 2011).

\section{LAS REPRESENTACIONES SOCIALES COMO EJE TEÓRICO}

Una de las principales características y, a la vez, el más grande reto de la "exclusión social", es su carácter polisémico (Sawaia, 2001); por tanto, es preciso señalar que si bien es necesaria una exploración y conocimiento de los procesos sociopolíticos y económicos para acercarse a posibles causas y crear sistemas de prevención, también es fundamental conocer los factores culturales, contextuales, imaginarios y simbólicos que acentúan e intensifican los procesos de exclusión (Álvarez de Hétier, 2001; Arruda, 2003). Por otro lado, como lo menciona Jodelet (2001), es en el campo de la psicología social que existe un nivel único de exploración que da sentido y un mayor entendimiento a este tipo de fenómenos. El abordaje de la "exclusión social" desde esta área permite el entendimiento de las ideas, símbolos y procesos cognitivos y psicológicos que incluyen las emociones, las cuales articulan los fundamentos materiales de esas relaciones, e intenta comprender de qué manera las personas o grupos son objeto de una distinción y así constituyen una categoría aparte.

Ante las necesidades diferentes de los fenómenos actuales y complejos surge, desde el seno de la psicología social, la teoría de las representaciones sociales que hace una ruptura epistemológica con el positivismo dominante (Arruda, 2003; Flores, 2010).
Las representaciones sociales (Rs) integran la información, actitud, imágenes, ideas y comportamientos respecto a un objeto determinado. Dicha representación no es homogénea, ya que existe siempre en relación con un contexto social específico o, más bien, con las características particulares de un grupo social.

Otra característica fundamental de las Rs (Rouquette \& Rateau, 1998; Flament \& Rouquette, 2003), es su carácter histórico, es decir, que éstas son producto de la historia y participan al mismo tiempo en su transformación. De igual forma, una Rs es colectivamente producida por un proceso global de comunicación (intercambios interindividuales y exposición a la comunicación de masas).

En este sentido, el concepto "niños de calle", en tanto derivado del fenómeno de la "exclusión social" (que interpela la cotidianidad de muchos citadinos y tiene una relevancia social y cultural), constituye un objeto de Rs. Muchas son las interrogantes, emociones y posturas que se generan en torno a la identidad de niños y jóvenes que viven o subsisten en la calle y lo que ello implica en términos estéticos y morales para cada contexto. Explicaciones del sentido común intentan dar respuesta a esta situación, alimentadas por los múltiples discursos de diversos medios de comunicación, y esto es lo que constituye la base del conocimiento comprendido en las Rs.

La presente investigación se abordó desde la perspectiva estructural (Abric, 2004), la cual considera que la organización de una Rs tiene una característica específica y debe ser organizada alrededor de dos sistemas:

- Un sistema central (o núcleo central), que determina la organización y el significado de las Rs. Este sistema está asociado a valores y normas (condiciones históricas e ideológicas) y se caracteriza por su estabilidad. Es generador de sentido, es decir, que crea y transforma el significado de otros elementos. El sistema es estabilizador de la RS y, por extensión, de la identidad grupal (Tosoli, De Oliveira \& Pereira, 2008)

- Un sistema periférico fuertemente relacionado con el contexto inmediato de la Rs. Este sistema tiene tres funciones (Navarro \& Gaviria, 2009):1) la prescripción de las conductas que permiten al sujeto saber qué tipo de comportamiento es per- 
tinente o no en un contexto y situación social; 2) permite la adaptación de la Rs, es decir, la personalización y apropiación que hacen los sujetos de ella, y 3) funciona como un sistema de defensa del núcleo central frente a los cambios de un contexto social. Este sistema periférico permite el anclaje de la Rs en el grupo, dándole un carácter particular.

Por último, esta investigación recupera el carácter formador de las emociones. Para Banchs (1996), éstas son modos de reconocer que un objeto es relevante e importante, ya que expresan juicios evaluativos y compromisos con determinadas visiones de las cosas, a la vez que se movilizan en los grupos, que establecen y refuerzan los núcleos de significado de acciones, creencias y relaciones; las emociones ejercen entonces una función preponderante en la selección de informaciones y en el posicionamiento favorable o desfavorable frente a aquello que se considera objeto de representación.

\section{MÉTODO}

\section{Contexto}

El presente estudio, basado en las Rs, considera sumamente importantes las características históricas, sociales y culturales del contexto. Por ello, el desarrollo de esta investigación se planteó en dos ciudades con marcadas y puntuadas diferencias: la Ciudad de México y la Ciudad de Guadalajara. Por una parte, la primera es la capital política, económica y social del país, reconocida por sus políticas progresistas e incluyentes y su gran densidad de población. Por otro lado, la ciudad de Guadalajara, segunda ciudad en importancia, es reconocida en el ámbito internacional por su gran aporte a la identidad cultural nacional, tanto por sus costumbres como por sus tradiciones, que se mantienen principalmente por el carácter conservador de su sociedad y sus integrantes.

\section{Escenario y participantes}

El escenario para la aplicación del instrumento fue la zona centro de ambas ciudades, que se distinguen por el alto índice de tránsito, comercio y actividad de poblaciones callejeras, de tal manera que la muestra estuvo compuesta por integrantes de las poblaciones callejeras, vendedores ambulantes, policías, miembros de diversas ONG, profesionistas, estudiantes y trabajadores de la zona, los cuales fueron seleccionados con el único requisito de nivel de cercanía al fenómeno del callejerismo. En el presente estudio participaron 110 personas: 56 de la Ciudad de México y 54 de la Ciudad de Guadalajara, de las cuales 63 fueron hombres y $47 \mathrm{mu}$ jeres, con un rango de edad que iba de 13 a 67 años.

\section{Procedimiento}

Para la obtención de datos se utilizó la evocación libre, la cual se presenta como una técnica privilegiada en la recolección de elementos que constituyen el contenido de una Rs (De Rosa, 2005; Vergès, 1994). La hipótesis de partida de esta técnica de recolección de información es la existencia de un funcionamiento cognitivo, a partir del cual "algunos términos son inmediatamente movilizados para expresar una representación" (Vergès, 1994, p. 235), siendo el carácter espontáneo y proyectivo de esta técnica, lo que permite acceder a los elementos que hacen parte del universo semántico del objeto estudiado, permiten identificar el núcleo central y el sistema periférico de las representaciones sociales.

El término inductor para la recolección de evocaciones libres fue la frase "Un niño de calle es...", a la cual los sujetos debían asociar un mínimo de tres palabras o expresiones. Se codificaron las respuestas con base en el orden de aparición. Para el tratamiento de los datos recolectados se utilizó el programa Evoc (Ensemble de programmes pemettant l'analyse des evoctions) versión 2000. Éste permite la ejecución de un análisis de tipo lexicológico, el cual calcula la frecuencia simple de cada palabra evocada, las frecuencias de cada palabra por jerarquías y la media de las órdenes en las evocaciones, para de ese modo organizar los términos encontrados según jerarquías (Tosoli, De Oliveira, \& Pereira, 2008). Con base en las respuestas, se obtuvieron elementos susceptibles de pertenecer al núcleo central y se definieron las diferentes periferias. El producto de las evocaciones fue organizado previamente, con lo que se conformó un corpus para el análisis, manteniendo el orden natural de las evocaciones de los sujetos. Finalmente, se generó un cuadro de cuatro divisiones con las siguientes propiedades. 
Tabla 1. Cuadro para las cuatro divisiones y sus propiedades

\begin{tabular}{l|l}
\hline 1er. cuadrante (núcleo central) & 2o. cuadrante (1a. periferia) \\
\hline $\begin{array}{l}\text { Agrupa los elementos más frecuentes y más importantes. } \\
\text { Está ligado a la memoria colectiva y a la historia del grupo. }\end{array}$ & Son encontrados los elementos periféricos más importantes. \\
\hline 3er. cuadrante (elementos de contraste) & 4o. cuadrante (2a. periferia) \\
\hline $\begin{array}{l}\text { Son encontrados los elementos con baja frecuencia, pero } \\
\text { considerados importantes por los sujetos. }\end{array}$ & $\begin{array}{l}\text { Es construida por los elementos menos frecuentes y menos } \\
\text { importantes, sin embargo, están más próximos del cotidiano. }\end{array}$ \\
\hline
\end{tabular}

Estos indicadores dan dos tipos de información diferente: por un lado, una dimensión colectiva, ya que se trata de términos fuertemente consensuales, por otro lado, una dimensión individual, ya que se trata de una distribución estadística hecha sobre la base del orden establecido por los sujetos (Chugar \& De Oliveira, 2008; Da Silva \& Chaves, 2009). Con fundamento en estos resultados, se realizó un análisis de categorías que puso en evidencia los temas o las unidades de sentido que organizan las Rs para el grupo.

Por último, se prosiguió con la utilización del programa Evoc para establecer una comparación léxica entre los dos contextos, y se recuperaron para esta investigación las palabras únicas de cada contexto evocadas con más alta frecuencia, de igual manera, las palabras de mayor evocación compartidas, es decir, consensuadas por ambos; esto se hizo con la finalidad de determinar elementos periféricos y centralidades determinadas por el contexto.

\section{RESULTADOS}

El análisis del corpus formado por las evocaciones de los sujetos, mostró que fueron un total de 1025 palabras registradas, que después de una depuración sistemática por similitud y eliminación de pronombres, artículos y preposiciones dio un total de 140 palabras evocadas 395 veces. Con el primer corpus se realizó un análisis lexicográfico de toda la muestra, el cual develó el prototipo de la Rs según la técnica Vergès (1994), descrita anteriormente, con el fin de reconocer la jerarquía de los elementos que la componen y plantear una hipótesis sobre su estructura (organización), que así permite comprender el sentido particular que tiene la Rs (vid. tabla 2).

Tabla 2. Resultados

\begin{tabular}{|c|c|}
\hline 1er. cuadrante (núcleo central) & 2o. cuadrante (1a. periferia) \\
\hline $\begin{array}{l}\text { personas-que-viven-en-la-calle } \\
\text { pobreza } \\
\text { sin-familia } \\
\text { tristeza }\end{array}$ & $\begin{array}{l}\text { falta-de-amor } \\
\text { flojos } \\
\text { necesitan-ayuda } \\
\text { niños-que-trabajan-en-la-calle } \\
\text { sin-educación } \\
\text { son-iguales-a-nosotros } \\
\text { vicios-adicciones }\end{array}$ \\
\hline 3er. cuadrante (elementos de contraste) & 4o. cuadrante ( $2 a$. periferia) \\
\hline $\begin{array}{l}\text { culpa-de-la-sociedad } \\
\text { deprimen } \\
\text { echan-desmadre } \\
\text { nada } \\
\text { problemas-familiares } \\
\text { soledad } \\
\text { víctimas }\end{array}$ & $\begin{array}{l}\text { con-problemas } \\
\text { desamparados } \\
\text { niños-irresponsables } \\
\text { no-quieren-salir-adelante } \\
\text { problemáticos } \\
\text { sufren }\end{array}$ \\
\hline
\end{tabular}


Por último, los resultados obtenidos en la comparación de contextos se exponen en la tabla 3.

\section{DISCUSIÓN}

Con base en los resultados obtenidos, el núcleo central está compuesto por cuatro conceptos, siendo "persona que vive en la calle" y "sin familia" dos creencias que permanecen hegemónicas en diversas investigaciones a escala global, atributos sumamente difundidos por los medios masivos de comunicación. Como ejemplo de ello, están las investigaciones efectuadas por Shaw (2002; 2002a), Llorens et al. (2005), Pojomovsky (2008), Navarro \& Gaviria (2010), por mencionar algunos.

Ante la evocación "persona que vive en la calle", se debe señalar que en el año 2000 se publicó el último estudio estadístico realizado por el DIF-UNICEF-DF sobre niños y jóvenes callejeros en la Ciudad de México, el cual contabilizó 14322 niños, niñas y adolescentes que usan las calles y otros espacios públicos de la urbe como lugares de trabajo y vivienda; sin embargo, de este universo total, sólo 7\% declaró vivir en la calle. De igual modo, en la experiencia del trabajo de calle en
Guadalajara, se obtuvieron datos cualitativos respecto a la constante y violenta persecución policiaca y acciones de limpieza social gubernamentales (Reza, 2011), las cuales tenían como consecuencia que muchos sujetos con los que se tuvo contacto buscaran múltiples alternativas para no pernoctar en la calle, por el riesgo que representa esta práctica, ya sea rentando cuartos de hoteles, vecindades o la toma de las conocidas "casonas" (casas abandonadas), lo que muchas veces representaba mayor riesgo por el hacinamiento y otras problemáticas relacionadas con la exclusión.

Prosiguiendo con el análisis, la alta frecuencia del concepto "personas que viven en la calle", como evocación a "niño de calle" muestra que la población en general abre un espectro mayor en el rango de edad al concepto, es decir, un "niño de calle" no es estrictamente un "niño", idea que parecería contradictoria conceptualmente, pero que tiene sentido en la percepción social, teniendo en cuenta otro dato significativo que arroja el censo elaborado por DIF-UNICEF-DF (2000): la población que vive en la calle está compuesta mayoritariamente por jóvenes adolescentes, que en $79 \%$ tiene más de 14 años; esto reforzaría lo planteado

Tabla 3. Resultados

\begin{tabular}{|c|c|c|}
\hline $\begin{array}{l}\text { Palabras únicas del contexto } \\
\text { "Ciudad de México" }\end{array}$ & $\begin{array}{c}\text { Palabras únicas del contexto } \\
\text { "Guadalajara" }\end{array}$ & $\begin{array}{c}\text { Palabras compartidas en ambos } \\
\text { contextos }\end{array}$ \\
\hline $\begin{array}{l}\cdot \text { flojos } \\
\cdot \text { falta-de-oportunidades } \\
\cdot \text { no-quieren-salir-adelante } \\
\cdot \text { personas-que-viven-en-la-calle } \\
\cdot \text { son-iguales-a-nosotros } \\
\cdot \text { piden-dinero } \\
\cdot \text { se-quejan-mucho } \\
\text { - buscan-integrarse-a-la-sociedad } \\
\cdot \text { falta-de-protección } \\
\cdot \text { necesitan-alguien-que-se-ocupe- } \\
\text { de-ellos } \\
\cdot \text { culpa-del-gobierno } \\
\text { - no-son-niños } \\
\text { rechazados-por-la-sociedad }\end{array}$ & 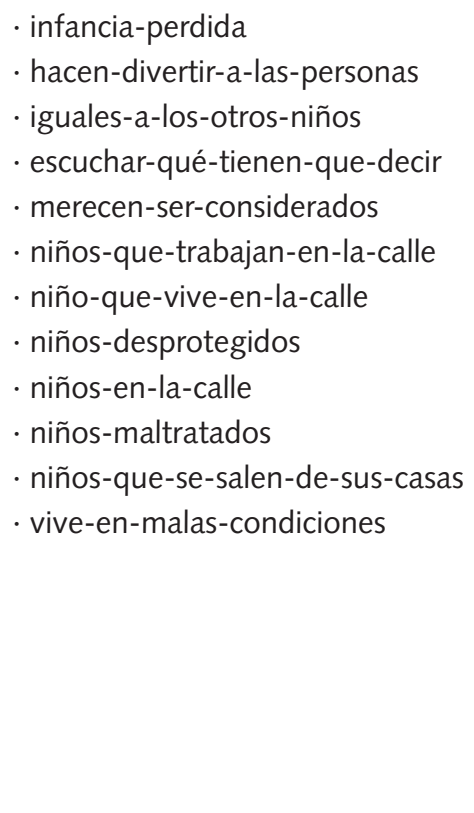 & $\begin{array}{l}\cdot \text { pobreza } \\
\cdot \text { vicios-adicciones } \\
\cdot \text { necesitan-ayuda } \\
\cdot \text { sin-educación } \\
\cdot \text { tristeza } \\
\cdot \text { falta-de-amor } \\
\cdot \text { sin-familia } \\
\cdot \text { sufren } \\
\cdot \text { carencias } \\
\cdot \text { con-problemas } \\
\cdot \text { culpa-de-la-sociedad } \\
\cdot \text { deprimen } \\
\cdot \text { echan-desmadre } \\
\cdot \text { niños-irresponsables } \\
\cdot \text { problemas-familiares } \\
\cdot \text { problemáticos } \\
\cdot \text { soledad } \\
\cdot \text { víctimas }\end{array}$ \\
\hline
\end{tabular}


anteriormente en esta investigación respecto al concepto "niño de calle", que ha dejado de ser exclusivo de niños y niñas en situación de calle.

Por otra parte, el atributo y creencia de "sin-familia” está totalmente ligado a los atributos periféricos, de "soledad" y "falta de amor". Ante esto, vale la pena hacer un análisis cualitativo al fenómeno del callejerismo, que está inmerso en una cultura de intercambios, relaciones y emociones. Para Makowski (2011), Strickland (2012), Pérez (2013a, 2013b), Pérez (2003), entre otros, estos factores son fundamentales y muy poco abordados para el entendimiento del arraigo a la cultura callejera, ya que muchas personas que salen a las calles re-configuran relaciones y lazos anteriormente perdidos, fortaleciendo nuevos vínculos e, indudablemente, crean nuevas estructuras. Así pues, en la actualidad no es extraño encontrar familias completas viviendo y subsistiendo en la calle; niños y jóvenes hijos de los primeros sujetos llamados "niños de calle" e incluso, una tercera generación de éstos (CDHDF, 2010; Barragán, 2010). Se ejemplifica esto en palabras de una mujer entrevistada para esta investigación, quien lleva 10 años viviendo en las calles de la Ciudad de México:

[...] Soy yo, mi familia, mis hijos... porque todos los que estamos aquí somos una familia, aunque no somos hijos de la misma mamá, como si lo fuéramos, ¿no?, nos echamos la mano en las buenas y en las malas... es lo que la gente no entiende cuando nos quieren llevar a los anexos, lo que menos queremos es que nos separen $[\ldots]$

Para finalizar este punto, cabe resaltar que las dos características atribuidas coinciden al pie de la letra con la definición dada por la UNICEF y citada al inicio de esta investigación respecto a la definición de "niño de calle", lo que confirma la solidez del núcleo central y demuestra las propiedades hegemónicas y poco mutables dentro de la Rs, creencias que se mantienen arraigadas al discurso cotidiano, a pesar de la disociación actual con el discurso científico. En esta forma, los elementos presentes en este núcleo dan un sentido fundamental e inflexible.

Un tercer concepto dentro del núcleo central es "pobreza", la cual más que un fenómeno causal, hace referencia a la condición socioeconómica propia de aquellos que viven en la calle y la posición de vulnerabilidad que dicha situación conlleva.

El último concepto dentro del núcleo central es "tristeza", que reafirma desde el marco teórico de las Rs, la importancia y función de las emociones ante un objeto social, y es la evocación de dicha emoción en la relación objeto-sujeto, la que dota de direccionalidad y significado (Banchs, 1996). La importancia de la movilización de emociones es una estrategia fundamental en la obtención de recursos materiales y simbólicos, tanto por parte de las poblaciones callejeras como de las organizaciones alrededor de éstas; ejemplo de ello son las prácticas de "charoleo", "palabreo" y "faquireo"3 de los sujetos callejeros y la mayoría de campañas mediáticas de las ONG, que por lo general, intensifican y exageran los valores negativos y la posición de víctimas arraigadas en la Rs del callejero, en aras de movilizar emociones que reditúen en apoyos y recursos (Magazine, 2007; Makowski, 2011; Pojomovsky, 2008).

Por último, el análisis comparativo entre contextos realizado por el programa Evoc ( $v i d$. figura 3 ) respecto a la evocación de palabras y su exclusividad por grupos, arroja las diferencias específicas entre los escenarios en la Rs del objeto social "niño de calle", diferencias que, como se mencionó antes, son determinadas por las características culturales, sociales y la historicidad única de cada contexto, lo que dota de suficientes elementos de discusión ante las políticas e intervenciones homogeneizantes y positivistas dominantes del fenómeno. Con base en los resultados, se puede decir que a pesar de que los dos contextos comparten elementos de Rs, las diferencias grupales son determinantes. Por un lado, en el grupo de Guadalajara, la referencia a la figura social del infante es una constante (p. ej.: niño trabajador, infancia perdida, niños como los otros, etc.), posiblemente debida al carácter conservador, nulas políticas públicas (legales) sobre el fenómeno, poco desarrollo, crítica y difusión de conceptos alternos al "niño de calle", y más específicamente, a la persecución y nula tolerancia institucional y municipal de la iden-

\footnotetext{
${ }^{3}$ Estos términos son utilizados por las poblaciones callejeras y organizaciones para definir las actividades y prácticas más frecuentes, cuya finalidad es la obtención de dinero o alimentos en la calle, autobuses, o sistemas de transporte alternos; "charoleo" se refiere a pedir dinero o pasar "la charola"; "palabreo" significa contar una historia generalmente trágica que expone los peligros que representa la calle; "faquireo" es el acto de escupir fuego o acostarse sobre vidrios.
} 
tidad de jóvenes y adultos callejeros que, como menciona Fletes (2004), pareciera que dan mal aspecto a la ciudad.

En el caso de la Ciudad de México, si bien resaltan conceptos relacionados con la tolerancia y diversidad (p. ej.: son igual a nosotros, no son niños, falta de oportunidades, buscan integrarse...), también saltan a la vista aspectos negativos vinculados al asistencialismo y pasividad de la población (p. ej.: flojos, se quejan mucho, no quieren salir adelante, sólo piden dinero, necesitan que alguien se ocupe de ellos...), percepción vinculada posiblemente con las prácticas derivadas por el gran número de instituciones y organizaciones que asisten a estos grupos en la ciudad.

Conforme a los resultados, con base en la teoría y en concordancia con otros estudios similares que han explorado diversas expresiones de la exclusión social y su RS (Navarro \& Gaviria, 2010; Campos \& Rouquette, 2003; O'Sullivan, Banch \& España, 2005), se puede inferir que existe la necesidad social de categorizar, de reducir la complejidad, de darle forma a lo desconocido y extraño que cuestiona los ideales sociales. Este proceso de categorización de las personas o grupos se hace a través de una simplificación, es decir, de una reducción abusiva de las características negativas del objeto, permitiendo y justificando las generalizaciones, las cuales, a su vez, se perpetúan y arraigan dentro de un ciclo constante de exclusión que queda reflejada en

\section{REFERENCIAS}

Abric, J. C. (2004). Metodología de recolección de las representaciones sociales (trad. J. Dacosta Chevrel \& F. Flores Palacios). En J. C. Abric (Ed.). Prácticas sociales y representaciones (pp. 53-74). México: Ediciones Coyoacán.

Alvarez de Hétier, L. (2001). Exclusión social y representaciones sociales: el caso de los niños de la calle. Fermentum, 11(30), 69-85.

Arruda, A. (2003). Living is Dangerous: Research Challenges in Social Representations. Culture Psychology, 9(4), 339-359.

Banchs, M. A. (1996). El papel de la emoción en la construcción de las representaciones sociales: invitación para una reflexión teórica. Papers on social representations, 5(2), 113-125. la complejidad de un concepto de por sí ambiguo, que en su mayoría evoca incapacidad, acciones de responsabilidad y tutelaje, y desde su perspectiva crea una representación de vulnerabilidad y necesidad de asistencia.

Se concluye que el "niño de calle" no se resume en una categoría precisa que incluye a un grupo definido de niños y niñas con historias y características homogéneas, sino que es una categoría surgida de la interacción entre una realidad social de personas que habitan y subsisten en las calles y las construcciones que la sociedad y sus grupos han ido haciendo de esto, con base en su experiencia, historia, cultura y mirada particular. El concepto, más que una etiqueta clara que delimita una realidad definida, objetiviza y ancla una serie de realidades, creencias, actitudes y afectos muchas veces difusos, en una visión entendible y justificable para la sociedad en general, la cual vive bajo dinámicas de producción y control. El concepto justifica una serie de actos y estigmas ante un grupo social fuertemente vulnerado, perpetuando dinámicas e intercambios que más allá de permitir una inclusión social y cambio identitario, promueven la reproducción de prácticas violentas, asistenciales y excluyentes, las cuales conllevan una serie de repercusiones físicas y psicológicas para los callejeros circunscritos en la etiqueta de "niño de calle".

Barragán, R. (2010). Prácticas cotidianas de personas adultas jóvenes que viven en la Plaza Zarco (Ciudad de México). Revista Latinoamericana de Ciencias Sociales, Niñez y Juventud, 8(1), 411-437.

Campos, P. \& Rouquette, M. L. (2003). Abordagem estrutural e componente afetivo das representações sociais. Psicologia: Reflexão e Critica, 16(3), 433-445. CDHDF (2010). Derechos de los jóvenes. Capítulo 28 del Diagnóstico de Derechos Humanos del Distrtio Federal. México: Comisión Nacional de los Derechos Humanos.

Chugar, I. \& De Oliveira, M. (2008). Metodología para evaluación post-ocupación de viviendas populares. El caso de la Comunidad María Auxiliadora, Cochabamba-Bolivia. Revista Universitaria de Investigación y Diálogo Académico, 4(3). 
Da Silva, M. \& Chaves, E. (2009). Atividade Física e BemEstar na Velhice. Revista de Salud Pública, 11(2), 225-236.

De Rosa, A. (2005). "Rede Associativa”: Uma técnica para captar a estrutura, os conteúdos e os índices de polaridade, neutralidade e estereotipia dos campos semânticos relacionados com as Representações Sociais. En A. Moreira (Ed.). Perspectivas Teórico-metodológicas em Representações Sociais. João Pessoa: UfPB/Editora Universitária.

DIF-DF-UNICEF (2000). Estudio de niñas, niños y jóvenes trabajadores en el D.F. México.

Flament, C. \& Rouquette, M. L. (2003). Anatomie des idées ordinaires. Comment étudier les représentations sociales. Paris: Armand Colin.

Fletes, C. (2004). Asistencia social: alcances y limitaciones. Estudios Jaliscienses, 55.

Flores P., F. (2010). Representación social y género: una relación de sentido común. En N. Blazquez, F. Flores \& M. Ríos (Eds.). Investigación feminista: epistemología, metodología y representaciones sociales (pp. 339358). México: UnAM.

Galende, F. (2011). Los excluidos. Breve elogio de lo inaparente. En J. Osorio \& F. Victoriano (Eds.). Exclusiones. Reflexiones críticas sobre subalternidad, hegemonía y biopolítica. México: Anthropos.

Garza, C. (2009). ¿Alguien sabe cuántos son? Rayuela. Revista Iberoamericana sobre Niñez y Juventud en Lucha por sus Derechos, 1(129), 131.

Jodelet, D. (2002). Representações sociais: um domínio em expansão. Em D. Jodelet (Ed.). As Representações sociais (pp. 17-44). Rio de Janeiro: Eduerj.

Llorens, M., Alvarado, C., Hernández, N., Jaramillo, Ú., Romero, M. \& Souto, J. (2005). Niños con experiencia de vida en la calle. México: Paidós.

Lucchini, R. (1996). Niño de la calle: identidad, sociabilidad y droga. Barcelona: Los Libros de la Frontera.

Magazine, R. (2007). Los niños de la calle en la Ciudad de México: un marco alternativo para su estudio. En D. Robichaux. Familia y Diversidad en América Latina. Estudios de casos (pp. 239-254). Buenos Aires: Clacso.

Maia da N., S. \& Alves, T. (2004). O "Menino de Rua” entre o sombrio e a aberrância da exclusão social. Estudos de Psicologia, 21(3), 161-172.
Makowski, S. (2011). Infancias y juventudes callejeras en la Ciudad de México. Regiones, suplemento de Antropología, 46 (octubre-diciembre).

Navarro C., O. \& Gaviria, M. (2009). Representaciones sociales del habitante de la calle. Universitas Psychologica, 9(2), 345-355.

Navarro C., O. \& Gaviria L., M. (2010). Social Representations of Homeless People. Universitas Psychologica, 9(2), 345-355.

O'Sullivan, J., Banchs, M. A. \& España, L. (2005). Medios de comunicación, pobreza y representaciones. Temas de comunicación, 12, 91-116.

Osorio, J. \& Victoriano, F. (Eds.) (2011). Exclusiones: reflexiones críticas sobre subalternidad, hegemonía y biopolítica. México: Anthropos.

Ossa, S. \& Lowick-Russell, A. (2009). Personas en situación de calle: el desafío de incluirlos a todos. Recuperado de http://www.hogardecristo.cl/files/2009/07/personas _situacion_calle.pdf

Padilla, R. \& Fletes, R. Conversación sobre los niños de la calle. Recuperado de http://medios.udg.mx/printpdf/ 9196

Pérez, G. (2003). La infancia callejera: apuntes para reflexionar el fenómeno. Revista Española de Educación Comparada, 8, 1-30.

Pérez, L. \& Arteaga, M. (2009). Identidad y práctica profesionales del educador de calle en México. Revista Latinoamericana de Ciencias Sociales, Niñez y Juventud, 7(2), 887-905.

Pérez, R. (2013a). Aprendiendo a sobrevivir: el uso del comercio informal como estrategia de subsistencia en personas en situación de calle. Memoria del Seminario El ambulantaje en la Ciudad de México: estudios recientes. México: UNAM-CEMCA.

Pérez, R. (2013b). Vivir y sobrevivir en la Ciudad de México. México: Plaza y Valdes.

Pojomovsky, J. (2008). Cruzar la calle: niñez y adolesencia en las calles de la ciudad. Buenos Aires: Espacio Editorial.

Reza, G. (2011). Impugnan programa de limpieza social en Guadalajara. Proceso. Recuperado de http://www. proceso.com.mx/?p=270278

Rizo, L. (2006). ¿A qué llamamos exclusión social? Polis, Revista de la Universidad Bolivariana, 5(15). 
Rouquette, M. L. \& Rateau, P. (1998). Introduction à l'étude des représentations sociales. Grenoble: PUG.

Sawaia, B. (2001). Exclusão o inclusão perversa. Em B. Sawaia (Ed.), As artimanhas da Exclusão. Análise psicossocial e ética da desigualdade social. Petrópolis: Vozes.

Shaw, K. (2002). La globalización de la exclusión. Brasil: Universidad Federal de Pernambuco/Universidad de Brasilia.

Shaw, K. (2002a). Hacia una teoría general de la calle. Shine a light, la red internacional pro niños de la calle. Recuperado de www.cit-dr.org/sal/library.html

Strickland, R. D. (2012). Poblaciones callejeras: de la asistencia a la represión. Desacatos, 38, 105-120.

Taracena, R. (2010). Hacia una caracterización psicosocial del fenómeno de callejerización. Revista Latinoamericana de Ciencias Sociales, Niñez y Juventud, 8(1), 393-409.
Tosoli, G., De Oliveira, D. \& Pereira, C. (2008). Representaciones sociales del sistema único de salud en el municipio de Río de Janeiro, según el enfoque estructural. Revista Latino-Americana de Enfermagem, 16(1), 122-129.

Urcola, M. (2011). La figura del "niño de la calle" como emblema de la época. Regiones, suplemento de Antropología, 46(octubre-diciembre), 10-16.

Vergès, P. (1994). Approche du noyau central: propriétés quantitatives et structurales, (pp. 233-253). En C. Guimelli (Ed.). Structures et Transformation des Représentations Sociales. Paris: Delachaux et Niestlé (Collection Textes de base en sciences sociales).

World-Bank (2014). Prosperity for all. Ending extreme poverty. Washington, DC: World Bank Group. 